Article

\title{
CLINICAL PROFILE OF PATIENTS WITH CORONARY TORTUOSITY AND ITS RELATION WITH CORONARY ARTERY DISEASE
}

\author{
Ankush Gupta $^{1 *}$, Prashant Panda ${ }^{2}$, Yash P Sharma ${ }^{3}$, Ashwin Mahesh ${ }^{4}$, Prafull Sharma ${ }^{5}$, Nalin K \\ Mahesh $^{6}$ \\ 1 1. Assistant professor, Department of Medicine \& Cardiology, Base Hospital Delhi Cantt, New Delhi-10, India. \\ Email-drankushgupta@gmail.com \\ 2 Assistant professor, Department of cardiology, Advanced Cardiac Center, PGIMER Chandigarh, India. Email- \\ prashantpanda85@gmail.com \\ 3 Head of the Department, Department of cardiology, Advanced Cardiac Center, PGIMER Chandigarh, India. Email- \\ ypspgi@gmail.com \\ 4 Resident, Department of Medicine, Jaipur Golden Hospital, New Delhi, India. Email- \\ ashwinmahesh2012@gmail.com \\ 5 Associate professor, Department of Medicine \& Cardiology, Base Hospital Delhi Cantt, New Delhi-10, India. Email- \\ draprafullsharma@gmail.com \\ 6 Head of the Department, Department of Medicine \& Cardiology, Base Hospital Delhi Cantt, New Delhi-10, India. \\ Email-drnkmahesh@gmail.com
}

* $\quad$ Correspondence: drankushgupta@gmail.com; Tel.: +919592903488

\begin{abstract}
Background: Coronary tortuosity is a common angiographic finding. Scarce data is available on clinical profile of patients with coronary tortuosity (CT) and its relation with coronary artery disease (CAD). Method: A total 224 patients undergoing angiography for suspected CAD were included in the study. CT was defined by the presence of $\geq 3$ consecutive bends of $>45$ degree measured at end-diastole in an epicardial artery $\geq 2 \mathrm{~mm}$ in diameter. CT was present in $45(20.08 \%)$ patients in the study and another 45 patients without CT was randomly selected as control (NCT group). Clinical profile of CT and NCT group was compared. Results: Incidence of $\mathrm{CT}$ was significantly higher in females $(\mathrm{p}=0.000)$ and hypertensives $(\mathrm{p}=0.001)$ patients. $\mathrm{CT}$ was most commonly seen in Left circumflex coronary artery. Incidence of CAD was significantly lower in CT group as compare to NCT group (0.02). Risk factors for CAD was associated with reduced incidence of CT. Majority (88.46\%) patient with CT without CAD presented with chronic stable angina out of which $(65.21 \%)$ had an objective evidence of myocardial ischemia. Conclusion: CT is more commonly seen females and hypertensive patients. It has negative correlation with CAD. Risk factors of CAD do not predict CT. CT itself can lead to myocardial ischemia.
\end{abstract}

Keywords: coronary tortuosity; myocardial ischemia; coronary artery disease; chronic stable angina.

\section{Introduction}

Coronary artery disease (CAD) is the leading cause of mortality and morbidity worldwide [1,2]. The gold standard for diagnosis of CAD is coronary angiography. Coronary tortuosity (CT) is a common angiographic finding encountered by cardiologists. Its association with chronic pressure load and impaired left ventricular relaxation and possibly coronary ischemia has been suggested by some studies [3-7]. Tortuous coronaries could hamper the ventricular function and have been proposed as an indicator of the ventricular dysfunction [4]. CT is also associated with reversible myocardial perfusion defects and chronic stable angina [7, 8]. Patients with CT often suffer the exercise-induced chest pain that typically disappears at rest [3]. These clinical findings indicate that CT may hinder coronary blood supply. However, no clinical data directly supports it. Whether CT leads to a significant decrease in the coronary blood supply is still unknown. The relationship between CT and CAD is still debatable. Only limited data is available in the literature on clinical profile of patients having CT either in isolation or with CAD. This study was done to observe the prevalence of CT in patients undergoing coronary angiography. This study also compares the clinical profile of patients having coronary tortuosity with or without CAD. 


\section{Materials and Methods}

This is a prospective observational study conducted at department of Cardiology, Advanced Cardiac Center, Postgraduate Institution of Medical Education and Research (Chandigarh), a tertiary care center in North India (Institutional Approval ID of the study: 1Trg-PG-2014/2309-15; Ethical approval ID: INT/IEC/185). Patients undergoing coronary angiography for suspected coronary artery disease (CAD), from January 2015 to December 2015 were enrolled in the study. Patients below the age of 18 years, patients with congenital heart disease and patients with slow flow on coronary angiography were excluded from the study. Suspected CAD patients were those who presented with acute coronary syndrome, chronic stable angina or asymptomatic patients undergoing angiography to rule out CAD, like prior to valve surgery. Detailed history and examination was done in all the patients. All patients underwent biochemical investigations which included fasting blood sugar, lipid profile, serum uric acid, and blood urea and serum creatinine. 12 lead electrocardiography and detailed transthoracic echocardiography was carried in all patients. All patients underwent elective coronary angiography according to the Judkins technique. CAD was defined as more than $50 \%$ luminal narrowing in at least one main coronary artery. CT was evaluated on special angulations, left anterior descending artery (LAD) was assessed in right anterior oblique with cranial angulations, left circumflex artery (LCX) in left anterior oblique with caudal angulations and right coronary artery (RCA) in right anterior oblique. CT was defined by the presence of $\geq 3$ consecutive curvatures of more than 45 degree measured at end- diastole in a major epicardial coronary artery $\geq 2 \mathrm{~mm}$ in diameter (Figure 1). Coronary flow was objectively quantified by using the corrected thrombolysis in myocardial infarction (TIMI) frame count method. Patients with a corrected TIMI frame count greater than two standard deviations from the normal range for the particular vessel were considered as having slow coronary flow, while those who had a corrected TIMI frame count within two standard deviations of the normal range were classified as having normal coronary flow. Statistical analysis was done using SPSS version 17. Normally distributed measurable data was compared using Student's t-test; whereas non-normally distributed or ordinal data using the Mann-Whitney test. The measurable data was presented as Mean \pm SD; whereas the skewed data was presented as their Median. In addition, Logistic regression was applied to find the independent factors which are associated with the groups. The association of all the Categorical /Classified data with the two groups was analyzed with Chi-Square test or the Fisher's exact test, whichever is applicable. $\mathrm{P}$ value $<0.05$ was taken as the level of significance.

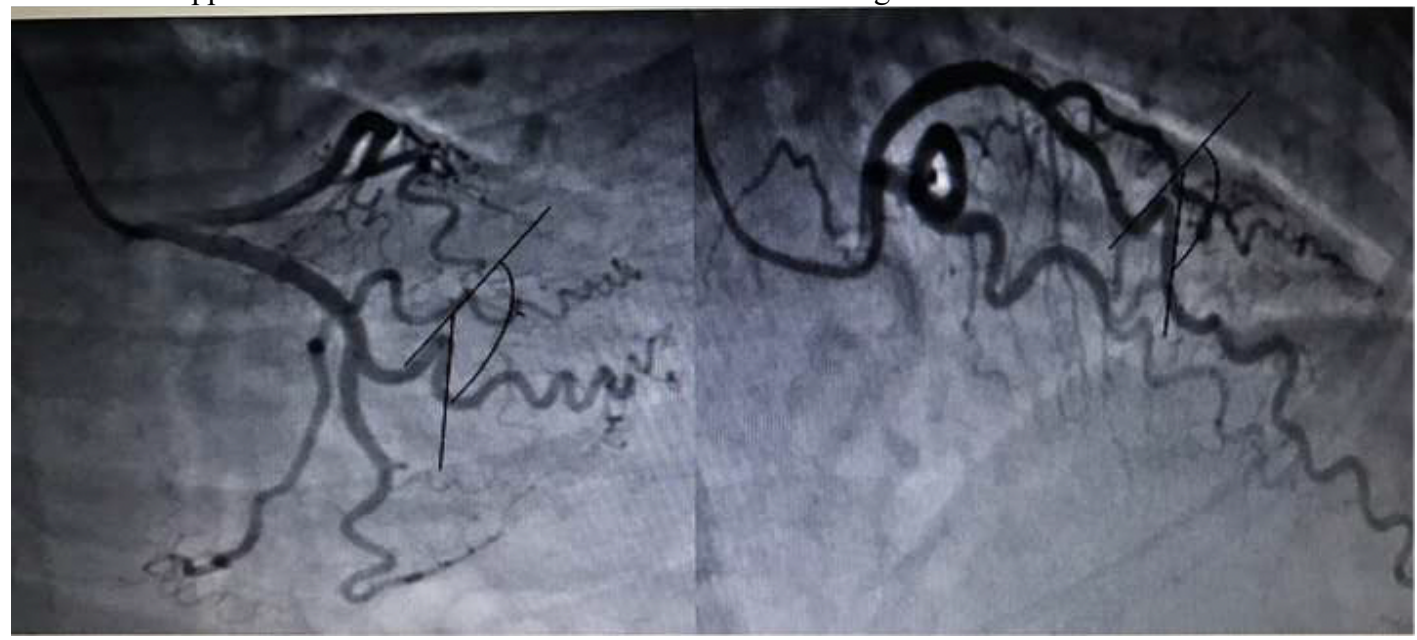

Figure 1: Images from two different patients showing coronary tortuosity with more than 45-degree bend.

\section{Results}

A total of 224 patients underwent angiography over a period of one year from January 2015 to December 2015. Out of which 45 patients had coronary tortuosity (20.08\%) (CT group), 45 more patients were randomly selected as control (NCT) group. In CT group 19(42.22\%) patients had CAD and 26 were without CAD (CT only). In NCT group 30((66.67\%)) patient had CAD and 15 patients had normal coronaries (Figure 2). The mean age of patients in the CT and NCT group was $57.38+9.43$ and $56.64+11.13$ years respectively. The 
number of male and female patients in the CT group was $16(35.6 \%)$ and 29(64.4\%) respectively. The number of male and female patients in NCT group was $37(82.2 \%)$ and $08(17.8 \%)$ respectively. Hypertension was present in $32(71 \%)$ patients in CT group and in $16(35.6 \%)$ patients in NCT group. The distribution of risk factors in CT and NCT group is shown in Table 1. CT incidence was significantly higher in females, hypertensive patients and patients with raised LDL. Incidence of CAD was significantly lower in CT group. Table 2 shows multivariate regression analysis using sex, hypertension (HTN), diabetes mellitus (DM), smoking, LDL and CAD to predict CT and NCT which was $87 \%$ and $91 \%$ respectively. So female gender, hypertension raised LDL were associated higher incidence of CT while diabetes, male sex, smoking, peripheral vascular disease (PVD), low HDL, reduced ejection fraction (EF) and CAD were linked with reduced incidence of CT. Left circumflex (LCX) was the most common single artery having CT. Out of 45 patients in CT group, 39(86.66\%) patients had CT in LCX artery either alone or in combination with other coronary artery, 26(57.77\%) had CT in left anterior descending (LAD) artery and 06(13.33\%) patients had CT in RCA (Table 3 ).

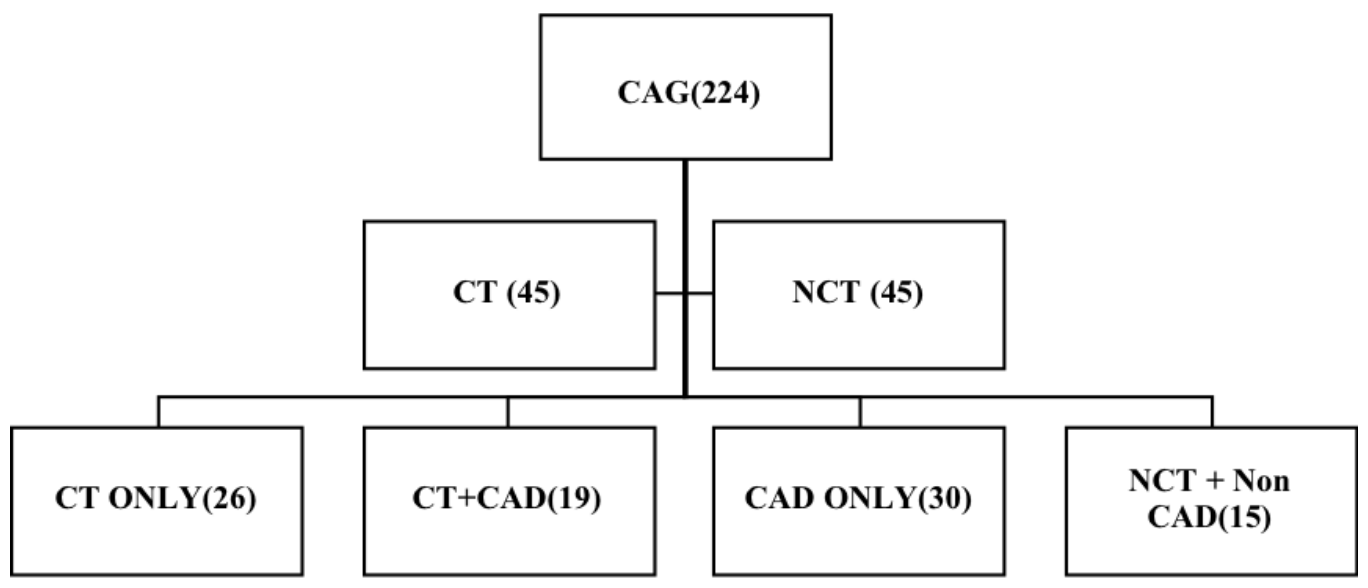

Figure 2: Flow chart showing distribution of study group patients into CT and NCT group and their further four subgroups. CAG- coronary angiography, CT- coronary tortuosity, NCT- noncoronary tortuosity, CAD- coronary artery disease.

Table 1 shows Clinical profile of patients in coronary tortuosity (CT) and non-coronary tortuosity group (NCT).

\begin{tabular}{|l|l|l|l|}
\hline Parameter & CT Group & NCT Group & p value \\
\hline AGE & $57.38 \pm 9.43$ & $56.64 \pm 11.13$ & 0.737 \\
\hline Female & $29(64.4)$ & $8(17.8)$ & 0.000 \\
\hline Male & $16(35.6)$ & $37(82.2)$ & 0.001 \\
\hline CAD & $19(42.22)$ & $30(66.67)$ & 0.02 \\
\hline HTN & $32(71.1)$ & $16(35.6)$ & 0.001 \\
\hline DM & $10(22.2)$ & $26(57.8)$ & 0.001 \\
\hline DL & $16(35.6)$ & $18(40)$ & 0.664 \\
\hline Smoking & $7(15.6)$ & $15(33.3)$ & 0.050 \\
\hline PVD & $0(0)$ & $9(20)$ & 0.002 \\
\hline
\end{tabular}




\begin{tabular}{|l|l|l|l|}
\hline $\begin{array}{l}\text { Family } \\
\text { History }\end{array}$ & $3(6.7)$ & $0(0)$ & 0.078 \\
\hline CVA & $0(0)$ & $0(0)$ & - \\
\hline HF & $6(13.3)$ & $6(13.3)$ & 1.000 \\
\hline Prior PCI & $1(2.2)$ & $2(4.4)$ & 0.557 \\
\hline Prior CABG & $0(0)$ & $0(0)$ & - \\
\hline SBP & $127.47 \pm 15.33$ & $117.73 \pm 22.6$ & 0.019 \\
\hline DBP & $80.71 \pm 8.18$ & $73.49 \pm 13.56$ & 0.003 \\
\hline Hb & $13.02 \pm 1.4$ & $12.79 \pm 0.92$ & 0.375 \\
\hline Creatinine & $0.89 \pm 0.25$ & $0.84 \pm 0.22$ & 0.350 \\
\hline LDL & $150.16 \pm 29.7$ & $126.53 \pm 39.25$ & 0.002 \\
\hline HDL & $49.22 \pm 8.24$ & $44.98 \pm 4.65$ & 0.004 \\
\hline TG & $164.91 \pm 37.58$ & $160.62 \pm 46.16$ & 0.630 \\
\hline FBS & $103.67 \pm 26.61$ & $126.73 \pm 37.26$ & 0.001 \\
\hline EF\% & $56.76 \pm 9.57$ & $45.04 \pm 13.37$ & 0.000 \\
\hline $\begin{array}{l}\text { Abnormal } \\
\text { ECG }\end{array}$ & $22(48.9 \%$ & $35(77.8 \%)$ & 0.004 \\
\hline
\end{tabular}

CAD- coronary artery disease, HTN-hypertension, DM- diabetes mellitus, DL dyslipidemia, PVD- peripheral vascular disease, CVA- cerebrovascular accident, HF- heart failure, PCI- percutaneous coronary intervention, CABG- coronary artery bypass graft, SBP- systolic blood pressure, DBP- diastolic blood pressure, Hb- haemoglobin, LDL- low density lipoprotein, HDL- high density lipoprotein, TG- triglycerides, FBS- fasting blood sugar, EF- ejection fraction.

Table 2: Multivariate Logistic regression of selected variable for prediction of CT.

\begin{tabular}{|l|l|l|l|l|l|l|l|}
\hline & B & S.E. & Wald & Df & Sig. & $\operatorname{Exp(B)}$ & 95\% C.I \\
\hline Male & -1.274 & .939 & 1.840 & 1 & .175 & .280 & $.044-1.762$ \\
\hline HTN absent & -1.445 & .815 & 3.142 & 1 & .076 & .236 & $.048-1.165$ \\
\hline Non Diabetic & 2.373 & .959 & 6.120 & 1 & .013 & 10.732 & $1.637-70.344$ \\
\hline Non smoker & 2.401 & 1.222 & 3.861 & 1 & .049 & 11.036 & 1.006 \\
& & & & & & & 121.054 \\
\hline Raised LDL & .070 & .021 & 11.723 & 1 & .001 & 1.073 & $1.031-1.117$ \\
\hline CAD absent & 1.380 & 1.244 & 1.232 & 1 & .267 & 3.977 & $.348-45.506$ \\
\hline
\end{tabular}

HTN- hypertension, LDL- low density lipoprotein, CAD- coronary artery disease.

Table 3 shows Distribution of Tortuosity in coronary arteries.

\begin{tabular}{|l|l|}
\hline ARTERY & FREQUENCY $(\mathbf{n}=\mathbf{4 5})$ \\
\hline LAD & $03(6.6 \%)$ \\
\hline LCX & $16(35.5 \%)$ \\
\hline
\end{tabular}




\begin{tabular}{|l|l|}
\hline LAD \& LCX & $19(42.2 \%)$ \\
\hline RCA & $01(2.2 \%)$ \\
\hline LAD \& RCA & $01(2.2 \%)$ \\
\hline LCX \& RCA & $01(2.2 \%)$ \\
\hline $\begin{array}{l}\text { LAD,LCX \& } \\
\text { RCA }\end{array}$ & $03(6.6 \%)$ \\
\hline
\end{tabular}

LAD- left anterior descending, LCX- left circumflex, RCA- right coronary artery.

\section{Discussion}

The prevalence of coronary tortuosity in the current study was $20.08 \%$. Study by Yang Li et al [9] and M. El Tahlawi et al [10] showed prevalence of $39.1 \%$ and $37.3 \%$ respectively. This difference was likely due to higher mean age in these studies as compared to current study. Advanced age has been associated with increased incidence of CT [11]. In present study the mean age of patients in CT group was $57.38 \pm 9.43$ years and females dominated the group with a prevalence of $64.4 \%$. While in the NCT group the mean age was $56.64 \pm 11.13$ years and males constituted $82.3 \%$ of this group. Female sex, hypertension and raised LDL was associated with increased incidence of CT. Hemodynamic forces have key role in modulation of vascular structure. Arteries may become tortuous due to increased pressure and reduced axial strain in an elastic cylindrical arterial model [12]. So close association between hypertension and CT is expected and CT might be one of the forms of arterial remodeling response to hypertension due to increased coronary pressure and blood flow. Increase incidence of $\mathrm{CT}$ in females as compared to males may be due to smaller cardiac size in females and coronary tortuosity decreases with cardiac enlargement [13]. Yang Li et al [9] and Groves et al [14] also found increased incidence of $\mathrm{CT}$ in females and hypertensive patients.

There have been conflicting reports regarding the correlation of coronary tortuosity and coronary artery disease. Previous studies have shown both negative $[9,14,15]$ and positive $[10,16,17]$ correlation between CT and CAD. In the present study we observed that in CT group $42.22 \%$ patients had CAD, whereas in NCT group $66.67 \%$ patients had CAD ( $\mathrm{p}=0.002$ ). So, we had a negative correlation between CT and CAD. Local hemodynamic factors like low shear stress plays an important role in the development of atherosclerosis and plaque stability through modulation of endothelial cell function and gene expression, while high shear stress is associated with protection from atherosclerosis [18]. So, the negative relationship between CT and CAD may be explained by the protective effect of high shear stress caused by CT. This may be one of the causes for lower incidence of CAD in female. In a study of coronary tortuosity by Türkmen S et al [15], a negative correlation between coronary tortuosity and severity of CAD was found. They used a novel numeric scoring system for quantitative assessment of CT. They defined the CT score as the sum of all bends on the main shafts of the major epicardial arteries. They found less CT in the males, smokers, diabetics and CAD patients. In a study by M El Tahlawi et al. [10], frequency of coronary tortuosity and coronary artery calcium (CAC) scoring was observed in patients with chronic stable angina using computed tomography coronary angiography. Definition used for CT was similar to the present study. They found higher CAC score in the CT group (p value $<0.05$ ) as compare to NCT group revealing that $\mathrm{CT}$ may be associated with subclinical atherosclerosis in the absence of obstructive CAD.

CT may be associated with the non- atherosclerotic cause of CAD like spontaneous coronary artery dissection (SCAD), especially in females. In a study of 246 patients of SCAD by Eleid MF [19], tortuosity was a common finding in patients presenting with their first SCAD episode. Tortuosity was most often observed in the left circumflex artery (LCX), followed by the left anterior descending (LAD), and the right coronary artery (RCA). In the present study most, patients had CT in left circumflex artery followed by LAD and RCA. Possible explanation for this higher prevalence of coronary tortuosity in LCX could be because of its natural course and possibly because of the higher hemodynamic stress being tackled by this artery.

In our study there were 26 patients, who had only coronary tortuosity. The mean age of patients with CT only was $55.31+8.8$ years. In this CT only group $69.2 \%$ were females and $73.2 \%$ patients were having hypertension. This is in agreement with previous studies which showed increased incidence of CT in females and hypertensive patients [9, 14, and 17]. Prevalence of diabetes mellitus, dyslipidemia, smokers and patients 
with heart failure was very low. Out of these 26 patients $23(88.5 \%)$ patients presented with chronic stable angina (CSA) and one patient had unstable angina. 12(46.1\%) patients had abnormal ECG at presentation. Out of 23 patients, who presented with chronic stable angina, 11 patients had positive treadmill test (TMT) for inducible ischemia, 2 patients showed reversible myocardial perfusion defect on stress myocardial perfusion imaging and 2 patients had abnormal regional wall motion abnormality on transthoracic echocardiography. So out of 23 patients presenting with chronic stable angina, we had 15 patients with objective evidence of myocardial ischemia but angiography showed only CT without significant coronary artery stenosis. There are few case reports in literature about coronary tortuosity as a cause of myocardial ischemia in patients with chronic stable angina. Zegers ES et al. [3] reported three cases of angina with abnormal stress test and angiography showing CT without fixed stenosis. They put forward the hypothesis that coronary tortuosity leads increase in energy loss resulting in a reduction in coronary perfusion pressure distal to CT segment, ultimately leading to ischemia [20]. Abdar Esfahani M et al. [21] reported that CT can cause CSA and found severity of clinical symptoms increase with severity of CT. DÖRTLEMEZ Ö et al [22] found significant decrease in systolic and diastolic coronary flow velocity distal to CT segment using intracoronary Doppler tipped guide wire. Yang Li et al. [23] found that compared to NCT, CT can result in more decrease of coronary blood pressure. They found more decrease in coronary pressure with increasing CT angle and number of CT curves. Xie X et al. [24] did a computational fluid dynamics (CFD) study to evaluate the impact of CT on the coronary blood supply. They concluded that CT has minor impact on coronary blood flow at rest; while during exercise, patients with CT had ineffective coronary auto-regulation and myocardial ischemia. One patient in CT only group presented with unstable angina, Chesnutt JK et al. [25] found that tortuosity of microvessel triggers platelet activation and thrombus formation. This may be responsible for acute coronary syndrome in CT only patient.

It is clear from the present study that $\mathrm{CT}$ without significant CAD produces clinical symptoms like chronic stable angina with objective evidence of myocardial ischemia. Present study showed negative correlation between CT and CAD. Previous studies have shown that CT may be associated with subclinical atherosclerosis $[10,17]$. We propose that patient with chronic stable angina and coronary tortuosity without significant CAD should be treated as CAD patients with antiplatelets, statins and antianginal drugs.

\section{Conclusions}

Coronary tortuosity is a common angiographic finding. Left circumflex is the most common coronary artery with tortuosity. Coronary tortuosity is commonly associated with females and hypertensive patients. Patients with coronary tortuosity have low incidence of CAD. Patients with coronary tortuosity can present with chronic stable angina and objective evidence of myocardial ischemia in the absence of significant obstructive coronary artery disease. Isolated coronary tortuosity in patients with chronic stable angina is often ignored and is labeled as normal coronaries. These patients remain symptomatic with angina with multiple health care visits, affecting their quality of life. These patients should be offered treatment with antiplatelets, statins and antianginal drugs to improve their quality of life.

\section{Clinical Implication}

Coronary artery tortuosity without significant obstructive coronary artery disease should be recognized as a cause of chronic stable angina. These patients should be given treatment with anteplatelets, statins and antianginals, which may result in marked improvement in symptoms, thereby improving the quality of life.

Supplementary Materials: Data sheet attached as a supplementary material.

Author Contributions: Conceptualization, Ankush Gupta and Yash Paul Sharma; Data curation, Ashwin Mahesh and Nalin Kumar Mahesh; Formal analysis, Ashwin Mahesh; Investigation, Ankush Gupta; Methodology, Prashant Panda and Ashwin Mahesh; Resources, Yash Paul Sharma; Supervision, Prashant Panda and Nalin Kumar Mahesh; Validation, Ankush Gupta, Prashant Panda and Yash Paul Sharma; Visualization, Yash Paul Sharma; Writing - original draft, Ankush Gupta and Prafull Sharma; Writing - review \& editing, Prafull Sharma and Nalin Kumar Mahesh.

Funding: "This research received no external funding".

Conflicts of Interest: "The authors declare no conflict of interest". 


\section{References}

1. Lloyd-Jones D, Adams RJ, Brown TM, et al. Heart disease and stroke statistics-2010 update A report from the American Heart Association. Circulation. 2010; 121(7): e46-215.

2. Zhang XH, Lu ZL, Liu L. Coronary heart disease in China. Heart. 2008;94(9):1126-31.

3. Zegers ES, Meursing BT, Zegers EB, Ophuis AO. Coronary tortuosity: a long and winding road. Netherlands Heart Journal. 2007;15(5):191-5.

4. Turgut O, Yilmaz A, Yalta K, et al. Tortuosity of coronary arteries: an indicator for impaired left ventricular relaxation? The international journal of cardiovascular imaging. 2007;23(6):671-7.

5. Jakob M, Spasojevic D, Krogmann ON, Wiher H, Hug R, Hess OM. Tortuosity of coronary arteries in chronic pressure and volume overload. Catheterization and cardiovascular diagnosis. 1996;38(1):25-31.

6. Gaibazzi N. Less than "straight" anginal symptoms. International journal of cardiology. 2011;148(3):385-6.

7. Gaibazzi N, Rigo F, Reverberi C. Severe coronary tortuosity or myocardial bridging in patients with chest pain, normal coronary arteries, and reversible myocardial perfusion defects. The American journal of cardiology. 2011;108(7):973-8.

8. Yang LI, Nai-feng LI, Zhong-ze GU, et al. Coronary tortuosity is associated with reversible myocardial perfusion defects in patients without coronary artery disease. Chin Med J. 2012;125(19):3581-3.

9. Li Y, Shen C, Ji Y, Feng Y, Ma G, Liu N. Clinical implication of coronary tortuosity in patients with coronary artery disease. PloS one. 2011;6(8): e24232.

10. El Tahlawi M, Sakrana A, Elmurr A, Gouda M, Tharwat M. The relation between coronary tortuosity and calcium score in patients with chronic stable angina and normal coronaries by CT angiography. Atherosclerosis. 2016;246:334-7.

11. Pancera P, Ribul M, Presciuttini B, Lechi A. Prevalence of carotid artery kinking in 590 consecutive subjects evaluated by Echo color doppler. Is there a correlation with arterial hypertension?. Journal of internal medicine. 2000;248(1):7-12.

12. Han HC. Twisted blood vessels: symptoms, etiology and biomechanical mechanisms. Journal of vascular research. 2012;49(3):185-97.

13. Hutchins GM, Bulkley BH, Miner MM, Boitnott JK. Correlation of age and heart weight with tortuosity and caliber of normal human coronary arteries. American heart journal. 1977;94(2):196-202.

14. Groves SS, Jain AC, Warden BE, Gharib W, Beto RJ. Severe coronary tortuosity and the relationship to significant coronary artery disease. West Virginia Medical Journal. 2009;105(4):14-8.

15. Serdar Turkmen, Zekeriya Kucukdurmaz, Caglar E Cagliyan, et al. Total tortuosity score: correlation with coronary artery disease and novel evaluation of possible risk factor. TGKD Cilt 17, Sayı kasım 2013:115121.

16. Cunningham KS, Gotlieb AI. The role of shear stress in the pathogenesis of atherosclerosis. Laboratory investigation. 2005;85(1):9-23.

17. Davutoglu V, Dogan A, Okumus S, et al. Coronary artery tortuosity: comparison with retinal arteries and carotid intima-media thickness. Kardiologia polska. 2012;71(11):1121-8.

18. Chatzizisis YS, Coskun AU, Jonas M, Edelman ER, Feldman CL, Stone PH. Role of endothelial shear stress in the natural history of coronary atherosclerosis and vascular remodeling: molecular, cellular, and vascular behavior. Journal of the American College of Cardiology. 2007;49(25):2379-93. 
19. Eleid MF, Guddeti RR, Tweet MS, et al. Coronary artery tortuosity in spontaneous coronary artery dissection angiographic characteristics and clinical implications. Circulation: Cardiovascular Interventions. 2014;7(5):656-62.

20. Gijsen FJ, Allanic E, Van de Vosse FN, Janssen JD. The influence of the non-Newtonian properties of blood on the flow in large arteries: unsteady flow in a 90 curved tube. Journal of biomechanics. 1999;32(7):705-13.

21. Abdar Esfahani M, Farzamnia H, Nezarat N. Chronic stable angina patients with tortuous coronary arteries: Clinical symptoms and risk factors. ARYA Atheroscler. 2012;7:S115-8.

22. DÖRTLEMEZ Ö, METIN M, ÇENGEL A, ALKAN M, CAYMAZ O, DÖRTLEMEZ H. Tortuosity of Coronary Arteries: A New Cause of Myocardial Ischemia. Gazi Medical Journal. 1993;4(3).

23. Li Y, Shi Z, Cai Y, et al. Impact of coronary tortuosity on coronary pressure: numerical simulation study. PloS one. 2012;7(8): e42558.

24. Xie X, Wang Y, Zhu H, Zhou H, Zhou J. Impact of coronary tortuosity on coronary blood supply: a patientspecific study. PloS one. 2013;8(5): e64564.

25. Chesnutt JK, Han HC. Tortuosity triggers platelet activation and thrombus formation in microvessels. Journal of biomechanical engineering. 2011;133(12):121004. 